\title{
Calidad del cuidado brindado por estudiantes de enfermería: experiencia y satisfacción del usuario
}

Quality of care provided by nursing students: patient experience and satisfaction

Qualidade dos cuidados oferecidos pelos estudantes de enfermagem: experiência e satisfação do usuário

Como citar este artículo: Ulloa Sabogal Iliana Milena, Valbuena Durán Luz Dalia, Camargo-Figuera Fabio Alberto. Calidad del cuidado brindado por estudiantes de enfermería: experiencia y satisfacción del usuario. Revista Cuidarte. 2021;12(1):e1130. http://dx.doi.org/10.15649/cuidarte.1130

Revista Cuidarte

doi) Rev Cuid. Ene. - Abril. 2021; 12(1): e1130 http://dx.doi.org/10.15649/cuidarte.1130

E-ISSN: 2346-3414

(1) Iliana Milena Ulloa Sabogal ${ }^{1}$

(1) Luz Dalia Valbuena-Durán²

(1) Fabio Alberto Camargo-Figuera ${ }^{3}$

1 Universidad Industrial de Santander, Escuela de Enfermería. Bucaramanga, Colombia.

E-mail:imulloa@uis.edu.co

2 Universidad Industrial de Santander, Escuela de Enfermería. Bucaramanga, Colombia.

E-mail: Idvaldur@correo.uis.edu.co

3 Universidad Industrial de Santander, Escuela de Enfermería. Bucaramanga, Colombia.

E-mail: falcamfi@uis.edu.co

\section{Resumen}

Introducción: El estudiante de enfermería, es un recurso importante en la evaluación del cuidado y la satisfacción percibida por los usuarios en las instituciones de salud en las que desarrollan sus prácticas formativas. Objetivo: Determinar la experiencia y satisfacción de la atención brindada por el estudiante de enfermería de la Universidad Industrial de Santander, desde la percepción de los usuarios hospitalizados en una institución pública prestadora de servicios de salud. Materiales y Métodos: Estudio descriptivo, transversal, cuantitativo, con una muestra de 217 usuarios hospitalizados en una institución de salud, a quienes se les aplicó el Cuestionario de Calidad del Cuidado de EnfermeríaCUCACE, versión en castellano. Se realizó un análisis descriptivo de la muestra y de los ítems del instrumento en las dimensiones satisfacción y experiencias con el cuidado, adicionalmente se evaluó la correlación entre los puntajes de las dimensiones. Resultados: La percepción de los usuarios en relación con la atención recibida por los estudiantes fue positiva en un $99.1 \%$, con una puntuación de 92.3 en la dimensión de satisfacción y de 88.4 en la dimensión de experiencias con el cuidado. El $66.2 \%$ de los usuarios describió que la atención no requiere ser mejorada. Conclusiones: Los usuarios refieren una percepción positiva en las dimensiones de experiencias y satisfacción del cuidado brindado por los estudiantes de enfermería, al percibir un cuidado individualizado, orientado y supervisado, basado en habilidades en educación, comunicación, humanización y conocimiento; lo que puede servir como un indicador de calidad de la atención al interior de las instituciones de salud y de los programas de enfermería.

Palabras clave: Atención de Enfermería; Calidad de la Atención de Salud; Estudiantes de Enfermería; Satisfacción del Paciente; Servicios de Salud.
Recibido: 20 de enero de 2020

Aceptado: 16 de agosto de 2020

Publicado: 12 de febrero de 2021 $\square *$ Correspondência

Iliana Milena Ulloa Sabogal

E-mail:imulloa@uis.edu.co 


\section{Quality of care provided by nursing students: patient experience and satisfaction}

\section{Abstract}

Introduction: Practicing nursing students are an essential resource in assessing patients' quality of care and satisfaction provided in healthcare institutions during their clinical practice. Objective: To determine patients' experience and satisfaction of care provided by practicing nursing students at the Industrial University of Santander from the perspective of patients who have been hospitalized in a healthcare center. Materials and Methods: A quantitative descriptive cross-sectional study was conducted with a sample of 217 patients who have been hospitalized in a healthcare center. Data were collected using the Nursing Care Quality Questionnaire (Spanish version). A descriptive analysis of the sample and instrument items was conducted in the patients' care satisfaction and experience dimensions. Results: Patients' perception of the care provided by nursing students was positive by $99.1 \%$, rating $92.3 \%$ in the care satisfaction dimension and $88.4 \%$ in the care experience dimension. $66.2 \%$ of patients reported that there was no need to improve care provision. Conclusions: Patients report having a positive perception of care experience and satisfaction provided by practicing nursing students as they perceive that individualized, guided and supervised care is provided on the basis of skills in education, communication, humanization and knowledge. The above can serve as a health care quality indicator in healthcare institutions and nursing programs.

Key words: Nursing Care; Quality of Health Care; Students Nursing; Patient Satisfaction; Health Services.

\section{Qualidade dos cuidados oferecidos pelos estudantes de enfermagem: experiência e satisfação do usuário}

\section{Resumo}

Introdução: $O$ estudante de enfermagem é um recurso importante na avaliação do cuidado e da satisfação percebida pelos usuários nas instituições de saúde onde eles desenvolvem suas práticas de formação. Objetivo: Determinar a experiência e satisfação do atendimento prestado pelo estudante de enfermagem da Universidade Industrial de Santander, com base na percepção dos usuários internados em uma instituição pública prestadora de serviços de saúde. Materiais e Métodos: Estudo descritivo, transversal, quantitativo, com uma amostra de 217 usuários internados em uma instituição de saúde, aos quais Ihes foi aplicado o Questionário de Qualidade do Cuidado de Enfermagem-CUCACE, versão em espanhol. Foi feita uma análise descritiva da amostra e dos itens do instrumento nas dimensões satisfação e experiências com o cuidado, além disso, foi avaliada a correlação entre as pontuações das dimensões. Resultados: A percepção dos usuários sobre o atendimento recebido pelos estudantes foi positiva em $99,1 \%$, com uma pontuação de 92,3 na dimensão satisfação e 88,4 na dimensão experiência com o cuidado. 66,2\% dos usuários descreveram que o atendimento não precisa ser melhorado. Conclusões: Os usuários relatam uma percepção positiva nas dimensões de experiências e satisfação do atendimento prestado pelos estudantes de enfermagem, pois percebem um cuidado individualizado, orientado e supervisionado, baseado em habilidades em educação, comunicação, humanização e conhecimento; o que pode servir como um indicador da qualidade do atendimento dentro das instituições de saúde e dos programas de enfermagem.

Palavras chaves: Cuidados de Enfermagem; Qualidade da Assitência á Saude; Estudantes de Enfermagem; Satisfação do Paciente; Serviços de Saúde. 


\section{Introducción}

La Calidad de la atención en salud se entiende "como la provisión de servicios de salud a los usuarios individuales y colectivos de manera accesible y equitativa a través de un nivel profesional óptimo, teniendo en cuenta el balance entre beneficios, riesgos y costos, con el propósito de lograr la adhesión y la satisfacción de los usuarios"1.

En Colombia, con el establecimiento de la Ley 100 de $1993^{2}$ y las reformas al sector salud mediante la Ley 1122 de $2007^{3}$, la Ley 1438 de $2011^{4}$ y el Decreto 1011 de 2006', se ha buscado garantizar la calidad en la prestación de los servicios de salud. Sin embargo, aún existen dificultades en la atención que inciden en la calidad y la satisfacción de los usuarios. Aunque dicha dificultad está relacionada con diversos factores, muchas veces recae en la labor que realizan los diferentes integrantes del equipo de salud, entre ellos el personal de enfermería, el cual ha sido considerado como el talento humano más requerido en el sistema de salud ${ }^{5}$.

Enfermería es un recurso fundamental y el eje insustituible alrededor del cual gira la totalidad de la atención en salud, debido a que es el personal que permanece más tiempo al cuidado de los pacientes, brindando asistencia técnica, emocional, afectiva, social y espiritual; aspectos que se ven reflejados en el grado de satisfacción de los usuarios y por consiguiente en la evaluación de la calidad asistencial ofrecida ${ }^{6,7}$.

La calidad de los servicios de enfermería se concibe como la atención oportuna, personalizada, humanizada, continua y eficiente que brinda el personal de enfermería, de acuerdo con estándares definidos para una práctica profesional competente y responsable, con el propósito de lograr la satisfacción del usuario y del prestador de servicios ${ }^{8}$.

Por lo tanto, brindar calidad en el cuidado es uno de los retos a los que se enfrenta enfermería, ya que sus características de ciencia humana le permiten preocuparse por el bienestar y la satisfacción de las necesidades y expectativas de las personas ${ }^{9}$. En este sentido, el grado de satisfacción del usuario, se ha convertido en un indicador esencial para evaluar la calidad, plantear acciones de mejora continua y alcanzar estándares de excelencia en la prestación de servicios de salud 5 .

En los últimos años, se han desarrollado varios estudios que a partir de diferentes instrumentos han evaluado el cuidado ofrecido por profesionales de Enfermería en pacientes hospitalizados; con resultados que demuestran un alto nivel de satisfacción de los usuarios en relación a la atención recibida ${ }^{5,6,8,10,11}$. Es de notar que la mayoría de investigaciones que miden la percepción del paciente en relación al cuidado de enfermería se fundamenta en el profesional más que en el estudiante.

En la búsqueda de la literatura disponible, a nivel nacional se encontraron los estudios de González ${ }^{12}$ y Campiño et al ${ }^{13}$ que evaluaron la percepción del paciente en relación al cuidado humanizado brindado por estudiantes de Enfermería utilizando el instrumento "Percepción de Comportamientos de Cuidado Humanizado de Enfermería (PCHE)". En el primer estudio los pacientes manifestaron que "siempre se percibe un cuidado humanizado" con un promedio de 120 puntos; las preguntas con los puntajes más bajos están relacionadas con instrucciones sobre el autocuidado, con un resultado de siempre $65 \%$, casi siempre $11 \%$, y nunca $20 \%$; y la identificación de necesidades de tipo físico, psicológico y espiritual donde el $64 \%$ expresó siempre, $16,9 \%$ casi siempre, $8,1 \%$ algunas veces y nunca $12,6 \%$. El segundo estudio presentó 
resultados de percepción de cuidado humanizado relativamente bajo ya que el 60,7\% manifestó que lo percibía "en algunas ocasiones", 17,9\%"nunca" y solo el 21,4\% "siempre.

En el contexto internacional, se han desarrollado estudios que han evaluado con diferentes escalas el nivel de satisfacción de los pacientes con la atención brindada por estudiantes de enfermería ${ }^{14-21}$ todos estos estudios en sus hallazgos coinciden en afirmar, que la participación del estudiante de enfermería en el cuidado del paciente, muestra un impacto positivo en el nivel de satisfacción de sus necesidades físicas, emocionales y de educación en salud, entre otras.

El estudiante de enfermería debe estar en capacidad de desarrollar lo aprendido dentro de sus procesos formativos enmarcados dentro los objetivos de formación de las escuelas de enfermería, sus referentes teóricos y modelos pedagógicos que vislumbran un sello particular en cada estudiante el proceso de formación ${ }^{13}$.

Por consiguiente, evaluar el cuidado que brinda el estudiante de enfermería también ha de convertirse en un indicador dentro del tema de la calidad, no sólo por el compromiso y responsabilidad con el usuario, la institución hospitalaria, la ética, las leyes y las normas de la profesión ${ }^{11}$, sino por su contribución en una autoevaluación permanente de las competencias curriculares orientadas a la formación de un profesional integral; comprometido con la salud y enfermedad de las personas y colectivos, que aporten en la construcción de sociedades saludables, tal como lo plantea la Misión del Programa de Enfermería de la Universidad Industrial de Santander 22.

En este sentido, la presente investigación tuvo como objetivo determinar la experiencia y satisfacción de la atención brindada por el estudiante de enfermería de la Universidad Industrial de Santander, desde la percepción de los usuarios en una institución pública universitaria prestadora de servicios de salud, Bucaramanga, Colombia.

\section{Materiales y métodos}

Estudio descriptivo de corte transversal con abordaje cuantitativo, realizado en los servicios de cirugía general, especialidades quirúrgicas (ortopedia-neurocirugía), medicina interna y ginecoobstetricia de una institución pública prestadora de servicios de salud integrales de mediana y alta complejidad de la ciudad de Bucaramanga, Colombia. La recolección de los datos fue realizada entre octubre de 2017 y octubre del 2018.

Los criterios de inclusión establecidos fueron: usuarios mayores de 18 años con una instancia hospitalaria igual o mayor a 48 horas; al cuidado de estudiantes del programa de enfermería de la Universidad Industrial de Santander durante la jornada de práctica formativa y en condiciones clínicas favorables para responder el instrumento de recolección de la información. Se excluyeron usuarios con alteraciones psiquiátricas, de comunicación, estado de conciencia (Glasgow menor de 15/15) o bajo efectos de sedación por medicamentos. 
En el cálculo del tamaño muestral se utilizó el programa Stata $12.1^{23}$. En la dimensión de experiencia con el cuidado, se calculó un puntaje promedio de 80, un poder del 80\%, un error tipo alpha del 5\%, una desviación estándar de 13 y una precisión (o error en la estimación) de 3 puntos para la media de la dimensión dando como resultado un tamaño de la muestra de 197 usuarios. Para la dimensión satisfacción en el cuidado se calculó un puntaje promedio esperado de 75 , un poder del $80 \%$, un error tipo al pha del 5\%, una desviación estándar de 20 y una precisión (o error en la estimación) de 4 puntos para la media de la dimensión obteniendo un tamaño de muestra de 148 usuarios. Las desviaciones estándar para las dimensiones de satisfacción y experiencias con el cuidado de enfermería, se establecieron según datos reportados en la literatura ${ }^{24}$. Finalmente se esperaba una tasa de respuesta del $90 \%$, para lo cual se estimó un tamaño final de la muestra para el presente estudio de 217 usuarios; seleccionados por medio de un muestreo por conveniencia.

El instrumento utilizado fue el Cuestionario de Calidad de Cuidados de Enfermería (CUCACE), versión en castellano validada por Alonso y cols ${ }^{25}$, de la versión original The Newcastle Satisfaction with Nursing Scales (NSNS). El cuestionario, en su versión original, muestra fiabilidad alfa de Cronbach de 0,92 para la "experiencia con los cuidados de enfermería"; y 0,97 para "satisfacción con los cuidados de enfermería"26. La validación al castellano realizada por Alonso y cols ${ }^{25}$ muestra fiabilidad alfa de Cronbach de 0,85 para la "experiencia con los cuidados de enfermería"; y 0,97 para la "satisfacción con los cuidados de enfermería", alcanzando un nivel de confiabilidad total de 0,90.

En Colombia, la versión en español fue adaptada y validada por primera vez en Bucaramanga. El resultado de fiabilidad mostró un alfa de Cronbach de 0,83 para la dimensión de "experiencia con los cuidados de enfermería", y 0,95 para la dimensión "satisfacción con los cuidados de enfermería", el índice de validez de contenido fue superior a 0,84 y los autores reportan un constructo válido ${ }^{24}$. También para este instrumento ha sido evaluada su reproducibilidad en el contexto regional, obteniendo aceptables coeficientes de correlación intraclase, por el orden de $0.7^{27}$.

Este cuestionario dividido en tres secciones: Variables sociodemográficas: nivel educativo, tipo de servicio, estrato socioeconómico y algunas preguntas de índole general. Las preguntas generales relacionadas con la atención recibida y la estancia en la unidad, presentan siete opciones de respuesta: Muy mala, Mala, Poca, Regular, Buena, Muy buena y Excelente. Experiencias con los cuidados de enfermería conformada por 26 preguntas, cada una con siete opciones de respuesta de 1-7(escala Likert), 14 formuladas en sentido positivo "completamente de acuerdo" y 12 en sentido negativo "completamente en desacuerdo". Es importante resaltar que las preguntas formuladas en sentido negativo deberán ser consideradas como positivas. Esta sección puntúa sobre 100, correspondiendo el máximo a la mejor atención posible. Satisfacción con los cuidados de enfermería consta de 19 preguntas todas en sentido positivo, cada una con 5 opciones de respuesta de 1-5 en la escala Likert: Muy satisfecho, Completamente satisfecho, Bastante satisfecho, Nada satisfecho y Poco satisfecho. Esta dimensión también puntúa sobre 100, y el máximo corresponde a la completa satisfacción. En el análisis de los datos las respuestas fueron recodificadas de 0 a 6, de acuerdo con la recomendación del manual de CUCACE. Las preguntas formuladas en las dimensiones de experiencias y satisfacción están orientadas a la opinión del usuario sobre la atención en el cuidado de enfermería; por lo cual se realizaron modificaciones mínimas en la redacción de algunos ítems y cambio con el término de "enfermera" por "estudiante de enfermería", con el fin de facilitar la comprensión de los usuarios durante su diligenciamiento. No se realizaron cambios en el orden ni exposición de las 
preguntas, de acuerdo con las sugerencias descritas en el manual del CUCACE.

La recolección de la información fue realizada por las enfermeras del equipo de investigación, quienes no mantuvieron relación con la coordinación o supervisión del grupo de estudiantes. A los usuarios que cumplían con los criterios de selección se les explicó el objetivo del estudio y se solicitó su participación voluntaria; se diligenció el consentimiento informado escrito y el cuestionario. Cada usuario fue entrevistado solo una vez y el diligenciamiento del cuestionario se llevó a cabo por las enfermeras investigadoras, con previo entrenamiento a partir de la utilización de un manual de procedimientos.

Los datos registrados en el cuestionario se sometieron a doble digitación en una base de datos en Microsoft Office Excel, con posterior contrastación de las posibles inconsistencias y corrección de errores de digitación. Para el análisis de los datos se utilizó el paquete estadístico Stata $12.1^{23}$. Se realizó un análisis descriptivo de la muestra, las variables cuantitativas se describieron con medidas de tendencia central de acuerdo con su distribución y las variables cualitativas con medidas de frecuencia absoluta y relativa. En cuanto a los resultados del instrumento CUCACE se describieron los resultados por ítem con frecuencias absolutas y relativas, para facilitar la interpretación de los resultados de CUCACE las opciones de respuesta de las preguntas de la dimensión de experiencia fueron agrupadas en tres categorías: positiva (Un poco de acuerdo, Muy de acuerdo, y Completamente de acuerdo), neutra (Ni de acuerdo ni en desacuerdo) y negativa (Un poco en desacuerdo, Muy en desacuerdo y Completamente en desacuerdo); la dimensión de satisfacción fue agrupada en tres niveles: positiva (Muy satisfecho y Completamente satisfecho), neutra (Bastante satisfecho) y negativa (Nada satisfecho y Poco satisfecho). Finalmente, para las preguntas generales relacionadas con la atención recibida y la estancia en la unidad fueron agrupadas en tres categorías: negativa (Muy mala, Mala y Poca), neutra (Regular) y positiva (Buena, Muy buena y Excelente).

Para evaluar la relación entre las dimensiones, primero se evaluó la normalidad de cada uno de los puntajes por medio de pruebas estadísticas (prueba de Shapiro-Wilk) y gráficas (Cajas y Bigotes), como dichas variables no presentaron una distribución normal se calculó un coeficiente de correlación de Spearman.

En esta investigación se tuvieron en cuenta los lineamientos de la Resolución 008430 del 4 de octubre de $1993^{28}$ y los principios éticos de la Ley 911 de $2004^{29}$, contó con el aval por parte del Comité de Ética en Investigación Científica de la Universidad Industrial de Santander (Acta No 17 de 2017) y del Comité Ética e Investigación de la Institución de Salud (Acta No 07 de 2017).

\section{Resultados}

\section{Características Sociodemográficas de los usuarios}

LaTabla 1, presenta el análisis del perfil sociodemográfico, el 54.4\% de los usuarios se encontraban en servicios de hospitalización médico-quirúrgicos y el $44.6 \%$ en Gineco-obstétricos, el género femenino tuvo la mayor representatividad de la población y la edad promedio fue de 39 años, estado civil en una relación de pareja estable. Con relación al nivel educativo, la mayoría de los usuarios manifestaron tener estudios de bachillerato completo; pertenecían a estratos socioeconómicos bajos; a nivel ocupacional desempeñan en un mayor porcentaje actividades independientes y otras como la dedicación al hogar. El tiempo de mayor estancia hospitalaria estuvo entre 2 y 3 días. 
Tabla 1. Caracterización del perfil sociodemográfico de los usuarios participantes del estudio. Bucaramanga, Colombia, 2017-2018

\begin{tabular}{|c|c|}
\hline Características sociodemográficas & n (\%) \\
\hline \multicolumn{2}{|l|}{ Sexo } \\
\hline Hombre & $90(41.5)$ \\
\hline Mujer & $127(58.5)$ \\
\hline \multicolumn{2}{|l|}{ Edad } \\
\hline Media - Mediana & $39.0-32(n: 217)$ \\
\hline$(D E-R I Q-M i n-M a ́ x)^{*}$ & $(19.2-62-18-89)$ \\
\hline \multicolumn{2}{|l|}{ Estado Civil } \\
\hline Soltero & $65(30.0)$ \\
\hline Unión libre & $96(44.2)$ \\
\hline Casado & $36(16.6)$ \\
\hline Divorciado/Viudo & $20(9.2)$ \\
\hline \multicolumn{2}{|l|}{ Escolaridad } \\
\hline Ninguna & $14(6.5)$ \\
\hline Primaria incompleta & $37(17.0)$ \\
\hline Primaria completa & $36(16.6)$ \\
\hline Bachillerato incompleto & 38 (17.5) \\
\hline Bachillerato completo & $64(29.5)$ \\
\hline Técnica o superior & 28 (12.9) \\
\hline \multicolumn{2}{|l|}{ Ocupación } \\
\hline Ninguna & $51(23.5)$ \\
\hline Independiente & 66 (30.4) \\
\hline Empleado & $26(12.0)$ \\
\hline Pensionado & $1(0.4)$ \\
\hline Otro & 73 (33.7) \\
\hline \multicolumn{2}{|l|}{ Estrato socioeconómico } \\
\hline 1 & $131(60.4)$ \\
\hline 2 & $69(31.8)$ \\
\hline 3 & $16(7.4)$ \\
\hline 4 & $1(0.4)$ \\
\hline \multicolumn{2}{|l|}{ Servicio } \\
\hline Puerperio & $49(22.6)$ \\
\hline Alto Riesgo Obstétrico & 40 (18.4) \\
\hline Sala de Partos & $10(4.6)$ \\
\hline Cirugía General & $36(16.6)$ \\
\hline Neurocirugía & $14(6.5)$ \\
\hline Medicina Interna & $53(24.4)$ \\
\hline Ortopedia & $15(6.9)$ \\
\hline \multicolumn{2}{|l|}{ Número de noches de estancia } \\
\hline $2-3$ & $100(46.1)$ \\
\hline $4-5$ & $22(10.2)$ \\
\hline $6-7$ & $25(11.5)$ \\
\hline $8-9$ & $20(9.2)$ \\
\hline 10 o más & $50(23.0)$ \\
\hline
\end{tabular}

Fuente: Diseño propio de los autores.

*DE: Desviación estándar, RIQ: Rango intercuartílico, Min: valor mínimo, Máx.: Valor máximo.

\section{Características en la atención recibida y estancia en la unidad}

En los resultados relacionados con las preguntas de índole general, se encontró una percepción en la que sobresale el tipo de respuesta "Buena" "Muy buena" y "Excelente". La percepción de los usuarios en relación con la atención recibida por los estudiantes fue positiva en un $99.1 \%$ y sólo el 33.8\% considera que dicha atención podría ser mejorada; es importante destacar que los participantes no presentaron percepciones negativas en la atención recibida por el estudiante de enfermería. En cuanto a la percepción de la atención recibida durante su estancia en el servicio hospitalario, fue positiva en un $95.9 \%$, como se muestra en la Tabla 2. Esto revela que en la mayoría de los usuarios se cumplieron sus expectativas frente al cuidado brindado por los estudiantes y por el personal del servicio. 
Tabla 2. Descripción de la atención recibida por los usuarios participantes del estudio. Bucaramanga, Colombia, 2017-2018

\begin{tabular}{lc}
\hline Característica & $\mathbf{n}(\%)$ \\
\hline $\begin{array}{l}\text { ¿Cómo valora usted la atención recibida por el estudiante de } \\
\text { enfermería en este servicio hospitalario? }\end{array}$ \\
$\quad$ Percepción Negativa & $0(0.0)$ \\
$\quad$ Percepción Neutra & $2(0.9)$ \\
$\quad$ Percepción Positiva & $215(99.1)$ \\
En su conjunto ¿Cómo valoraría su estancia en este Servicio & \\
Hospitalario? & \\
$\quad$ Percepción Negativa & $1(0.4)$ \\
$\quad$ Percepción Neutra & $8(3.7)$ \\
$\quad$ Percepción Positiva & $208(95.9)$ \\
¿La atención recibida por los estudiantes de enfermería podría ser & \\
mejorada en algúraspecto? * & \\
$\quad$ Si & $73(33.8)$ \\
$\quad$ No & $143(66.2)$ \\
\hline
\end{tabular}

Fuente: Diseño propio de los autores.

* Pregunta con una observación sin dato.

En el análisis de las respuestas registradas en las dimensiones de experiencias y satisfacción, se encontró que los usuarios dejaron algunas preguntas sin contestar o marcaban dos respuestas en una misma pregunta, lo que conllevó a no ser registrada en la base de datos y ser tratada como una respuesta invalidada.

\section{Experiencias con el cuidado del estudiante de enfermería}

La distribución de las respuestas en la escala de experiencias con el cuidado están presentadas en la Tabla 3. En general las respuestas evidencian puntajes por encima del $90 \%$ para una percepción positiva, lo que significa que la mayoría de los usuarios respondieron"completamente de acuerdo" en los ítems positivos y "completamente en desacuerdo" en los ítems negativos. Sin embargo, en las preguntas "El estudiante desconocía la condición de salud, que usted está padeciendo", y "El estudiante desconocía lo que sus otros compañeros estaban haciendo con otros pacientes", tuvieron una percepción positiva en un 65,9\% y 54,8 \% respectivamente. Este resultado debe tenerse en cuenta, en razón a que el conocimiento por parte del estudiante de la situación de salud del usuario y del trabajo que desarrollan sus compañeros juega un papel importante en la percepción de la experiencia del cuidado. Otro hallazgo de resaltar corresponde a la pregunta "Los médicos y el estudiante de enfermería trabajaron juntos como un equipo de trabajo", en la que se obtuvo tan sólo un $68.2 \%$ de percepción positiva, aspecto que demuestra la importancia que el estudiante de enfermería brinde cuidado en conjunto con el personal médico. 
Tabla 3. Descripción de las respuestas de los usuarios participantes en la dimensión de experiencias con los cuidados. Bucaramanga, Colombia, 2017-2018

\begin{tabular}{|c|c|c|c|}
\hline Ítem Dimensión: Experiencias con los cuidados & $\begin{array}{l}\text { Negativa } \\
\% \text { (n) }\end{array}$ & $\begin{array}{l}\text { Neutra } \\
\%(n)\end{array}$ & $\begin{array}{l}\text { Positiva } \\
\% \text { (n) }\end{array}$ \\
\hline Fue fácil relacionarse con el estudiante de enfermería & $1.4(3)$ & $0.9(2)$ & $97.7(212)$ \\
\hline $\begin{array}{l}\text { El estudiante de enfermería dio prioridad a sus cuidados sobre los } \\
\text { cuidados de otros pacientes }\end{array}$ & $0.5(1)$ & $1.4(3)$ & $98.1(213)$ \\
\hline $\begin{array}{l}\text { El estudiante de enfermería no le informó lo suficiente sobre su } \\
\text { condición de salud o tratamiento }\end{array}$ & 17.1(37) & $5.5(12)$ & $77.4(168)$ \\
\hline $\begin{array}{l}\text { El estudiante de enfermería se despreocupaba fácilmente de su } \\
\text { cuidado. }\end{array}$ & $7.4(16)$ & $0(0)$ & $92.6(201)$ \\
\hline $\begin{array}{l}\text { El estudiante de enfermería tardaba mucho tiempo en llegar } \\
\text { cuando usted le llamaba. }\end{array}$ & $6.5(14)$ & $0.9(2)$ & $92.6(201)$ \\
\hline $\begin{array}{l}\text { El estudiante de enfermería le brindaba información sólo } \\
\text { cuando usted la necesitaba. }\end{array}$ & $12.0(26)$ & $6.0(13)$ & $82.0(178)$ \\
\hline $\begin{array}{l}\text { El estudiante de enfermería no parecía saber la condición de } \\
\text { salud que usted está padeciendo. }\end{array}$ & 24. $4(53)$ & $9.7(21)$ & $65.9(143)$ \\
\hline $\begin{array}{l}\text { El estudiante de enfermería realizó actividades para mantener } \\
\text { su confort }\end{array}$ & $2.8(6)$ & $4.1(9)$ & $93.1(202)$ \\
\hline $\begin{array}{l}\text { El estudiante de enfermería le mandaba hacer cosas sin que usted } \\
\text { se encontrara preparado. }\end{array}$ & $1.8(4)$ & $1.8(4)$ & $96.4(208)$ \\
\hline $\begin{array}{l}\text { A pesar de lo ocupado que estaba el estudiante de enfermería, } \\
\text { buscaba tiempo para usted. }\end{array}$ & $3.7(8)$ & $0(0)$ & $96.3(209)$ \\
\hline Vio al estudiante de enfermería como un amigo(a). & $2.8(6)$ & $6.9(15)$ & $90.3(196)$ \\
\hline $\begin{array}{l}\text { El estudiante de enfermería pasaba más tiempo apoyando a otros } \\
\text { pacientes que se encontraban mal, que apoyándolo a usted. }\end{array}$ & $5.1(11)$ & $4.1(9)$ & $90.8(197)$ \\
\hline $\begin{array}{l}\text { El estudiante de enfermería le preguntaba regularmente cómo se } \\
\text { sentía para asegurarse de que usted estaba bien. }\end{array}$ & $2.3(5)$ & $0.9(2)$ & $96.8(210)$ \\
\hline El estudiante de enfermería permitía que la situación lo sobrepasara. & $6.9(15)$ & $1.9(4)$ & $91.2(197)$ \\
\hline El estudiante de enfermería no tenía interés en usted como persona. & $10.1(22)$ & $0(0)$ & $89.9(195)$ \\
\hline $\begin{array}{l}\text { El estudiante de enfermería le explicó lo que le está pasando con } \\
\text { relación a su situación de salud }\end{array}$ & $4.1(9)$ & $2.3(5)$ & $93.6(203)$ \\
\hline $\begin{array}{l}\text { El estudiante de enfermería le explicaba los procedimientos antes } \\
\text { de hacerlos }\end{array}$ & $0.9(2)$ & $2.8(6)$ & $96.3(209)$ \\
\hline $\begin{array}{l}\text { El estudiante de enfermería le decía a la profesora los cuidados } \\
\text { que le estaba brindando. }\end{array}$ & $3.2(7)$ & $9.2(20)$ & $87.6(190)$ \\
\hline $\begin{array}{l}\text { El estudiante de enfermería sabía qué hacer sin depender de } \\
\text { la profesora. }\end{array}$ & $9.2(20)$ & $3.2(7)$ & $87.6(190)$ \\
\hline $\begin{array}{l}\text { El estudiante de enfermería solía irse y olvidar lo que usted le } \\
\text { estaba pidiendo. }\end{array}$ & $2.8(6)$ & $0.9(2)$ & $96.3(209)$ \\
\hline $\begin{array}{l}\text { El estudiante de enfermería se aseguraba de que usted tuviera } \\
\text { intimidad cuando la necesitaba. }\end{array}$ & $3.2(7)$ & $10.1(22)$ & $86.6(188)$ \\
\hline $\begin{array}{l}\text { El estudiante de enfermería tenía tiempo para sentarse y hablar } \\
\text { con usted. }\end{array}$ & $5.1(11)$ & $5.1(11)$ & $89.8(195)$ \\
\hline $\begin{array}{l}\text { Los médicos y el estudiante de enfermería trabajaron juntos como } \\
\text { un equipo de trabajo. }\end{array}$ & $10.6(23)$ & $21.2(46)$ & $68.2(148)$ \\
\hline $\begin{array}{l}\text { El estudiante de enfermería no parecía saber lo que sus otros } \\
\text { compañeros estaban haciendo. }\end{array}$ & $21.2(46)$ & $24.0(52)$ & 54.8 (119) \\
\hline $\begin{array}{l}\text { El estudiante de enfermería sabía lo mejor que debía hacer en } \\
\text { cada momento. }\end{array}$ & $0.5(1)$ & $1.8(4)$ & $97.7(212)$ \\
\hline $\begin{array}{l}\text { Había un buen ambiente en la unidad gracias al estudiante de } \\
\text { enfermería. }\end{array}$ & $0.9(2)$ & $3.7(8)$ & 95.4 (207) \\
\hline
\end{tabular}

Fuente: Diseño propio de los autores. 


\section{Satisfacción con el cuidado del estudiante de enfermería}

El análisis de las respuestas de la escala de satisfacción, permitió conocer que la pregunta con mayor porcentaje para una satisfacción positiva fue"La manera en que el estudiante le explicaba las cosas" con un $97.7 \%$, seguida de las preguntas "La amabilidad del estudiante", "El trato que recibió del estudiante como persona" $y$ "El modo de hacer su trabajo el estudiante de enfermería" todas con un $97.2 \%$, puntajes que reflejan una práctica impregnada de valores y principios humanísticos por parte del estudiante de enfermería. Cabe resaltar que la pregunta "La forma como el estudiante de enfermería le informaba a su familia" obtuvo el mayor porcentaje de satisfacción negativa en un $6.0 \%$, lo cual podría estar relacionado con la falta de orientación del estudiante a los familiares acerca del estado de salud del usuario, el horario de ingreso de la visita o la no presencia de familiares durante el tiempo de la jornada de las prácticas formativas de los estudiantes en la institución de salud, conforme la Tabla 4.

\section{Tabla 4. Descripción de las respuestas de los usuarios participantes en la dimensión de satisfacción con los cuidados. Bucaramanga, Colombia, 2017-2018}

\begin{tabular}{|c|c|c|c|}
\hline Ítem Dimensión: Satisfacción con los cuidados & $\begin{array}{c}\text { Negativa } \\
\%(n)\end{array}$ & $\begin{array}{l}\text { Neutra } \\
\%(n)\end{array}$ & $\begin{array}{l}\text { Positiva } \\
\% \text { (n) }\end{array}$ \\
\hline $\begin{array}{l}\text { La cantidad de tiempo que el estudiante de enfermería pasó con } \\
\text { usted. }\end{array}$ & $0.9(2)$ & $8.8(19)$ & $90.3(196)$ \\
\hline $\begin{array}{l}\text { La capacitación del estudiante de enfermería para hacer bien su } \\
\text { trabajo. }\end{array}$ & $0.9(2)$ & $8.3(18)$ & $90.8(197)$ \\
\hline $\begin{array}{l}\text { El estudiante de enfermería estaba siempre cerca cuando usted } \\
\text { lo necesitaba. }\end{array}$ & $0.9(2)$ & $5.1(11)$ & $94.0(204)$ \\
\hline $\begin{array}{l}\text { El estudiante de enfermería conocía los cuidados que usted } \\
\text { requería. }\end{array}$ & $0.9(2)$ & $4.6(10)$ & $94.5(205)$ \\
\hline $\begin{array}{l}\text { La rapidez con la que el estudiante de enfermería acudía cuando } \\
\text { usted le llamaba. }\end{array}$ & $1.8(4)$ & $6.5(14)$ & $91.7(199)$ \\
\hline $\begin{array}{l}\text { La manera como le atendió el estudiante de enfermería le hizo } \\
\text { sentir como en casa. }\end{array}$ & $0.9(2)$ & $3.7(8)$ & $95.4(207)$ \\
\hline $\begin{array}{l}\text { La cantidad de información que el estudiante de enfermería le } \\
\text { brindó acerca de su situación de salud y tratamiento. }\end{array}$ & $1.8(4)$ & $6.0(13)$ & $92.2(200)$ \\
\hline $\begin{array}{l}\text { La frecuencia con que el estudiante de enfermería comprobaba } \\
\text { que usted se encontraba bien. }\end{array}$ & $1.4(3)$ & $5.1(11)$ & $93.5(203)$ \\
\hline La amabilidad del estudiante de enfermería. & $0(0)$ & $2.8(6)$ & $97.2(211)$ \\
\hline $\begin{array}{l}\text { La manera en que el estudiante de enfermería le explicaba las } \\
\text { cosas. }\end{array}$ & $0(0)$ & $2.3(5)$ & $97.7(212)$ \\
\hline $\begin{array}{l}\text { La forma como el estudiante de enfermería le informaba a su } \\
\text { familia. }\end{array}$ & $6.0(8)$ & $3.8(5)$ & $90.2(120)$ \\
\hline El modo de hacer su trabajo el estudiante de enfermería. & $0.9(2)$ & $1.8(4)$ & $97.2(211)$ \\
\hline $\begin{array}{l}\text { El tipo de información que el estudiante de enfermería le daba } \\
\text { acerca de su situación de salud y tratamiento. }\end{array}$ & $1.4(3)$ & $4.6(10)$ & $94.0(204)$ \\
\hline El trato que recibió del estudiante de enfermería como persona. & $0(0)$ & $2.8(6)$ & $97.2(211)$ \\
\hline $\begin{array}{l}\text { La forma como el estudiante de enfermería escuchaba sus } \\
\text { preocupaciones. }\end{array}$ & $0.9(2)$ & $4.2(9)$ & $94.9(204)$ \\
\hline $\begin{array}{l}\text { La autonomía que le dio el estudiante de enfermería para } \\
\text { participar en su propio cuidado. }\end{array}$ & $0.5(1)$ & $3.7(8)$ & $95.8(207)$ \\
\hline $\begin{array}{l}\text { La disponibilidad del estudiante de enfermería para responder a } \\
\text { sus necesidades de cuidado. }\end{array}$ & $0(0)$ & $4.2(9)$ & $95.8(208)$ \\
\hline $\begin{array}{l}\text { El grado de intimidad que le proporcionó el estudiante de } \\
\text { enfermería. }\end{array}$ & $0.5(1)$ & $3.8(8)$ & $95.7(203)$ \\
\hline $\begin{array}{l}\text { El conocimiento del estudiante de enfermería sobre sus } \\
\text { necesidades de cuidado. }\end{array}$ & $0.5(1)$ & $5.5(12)$ & $94.0(204)$ \\
\hline
\end{tabular}

Fuente: Diseño propio de los autores. 
En la Tabla 5 y Figura 1, se evidencia que los puntajes totales de cada una de las dos dimensiones del instrumento CUCACE, obtuvieron medias y medianas altas, indicando que los usuarios en general tuvieron una percepción positiva en la experiencia y satisfacción con los cuidados brindados por los estudiantes de enfermería. También se aprecia que existe correlación positiva moderada entre las dos dimensiones, en razón a que el coeficiente de correlación de Spearman fue de $r=$ 0.53 , lo cual indica que puntajes altos de la dimensión de experiencia se relaciona con puntajes altos de la dimensión de satisfacción.

Tabla 5. Correlación entre las dimensiones experiencias y satisfacción con los cuidados en los usuarios participantes. Bucaramanga, Colombia, 2017-2018

\begin{tabular}{lcc}
\hline Dimensión & Estadístico* & $\begin{array}{c}\text { Correlación* } \\
\text { (Valor de p) }\end{array}$ \\
\hline Satisfacción con los cuidados & Media $=92.3$ & $\mathrm{r}=0.53$ \\
& Mediana $=97.3$ & $\mathrm{DE}=0.001)$ \\
& $\mathrm{RIQ}=50$ & \\
& Min $=38.8$ & \\
Máx $=100$ & $\mathrm{SW}=\mathrm{p}<0.001$ \\
\hline Experiencias con los cuidados & Media $=88.4$ \\
& Mediana $=91.0$ \\
$\mathrm{DE}=9.13$ & $\mathrm{RIQ}=32.6$ \\
$\mathrm{Min}=52.6$ & $\mathrm{Máx}=100$ \\
$\mathrm{SW}=\mathrm{p}<0.001$
\end{tabular}

Fuente: Diseño propio de los autores.

* Coeficiente de correlación de Spearman, DE: Desviación estándar, RIQ: Rango intercuartílico, Min: valor mínimo, Máx: Valor máximo, SW = Prueba de normalidad de Shapiro-Wilk

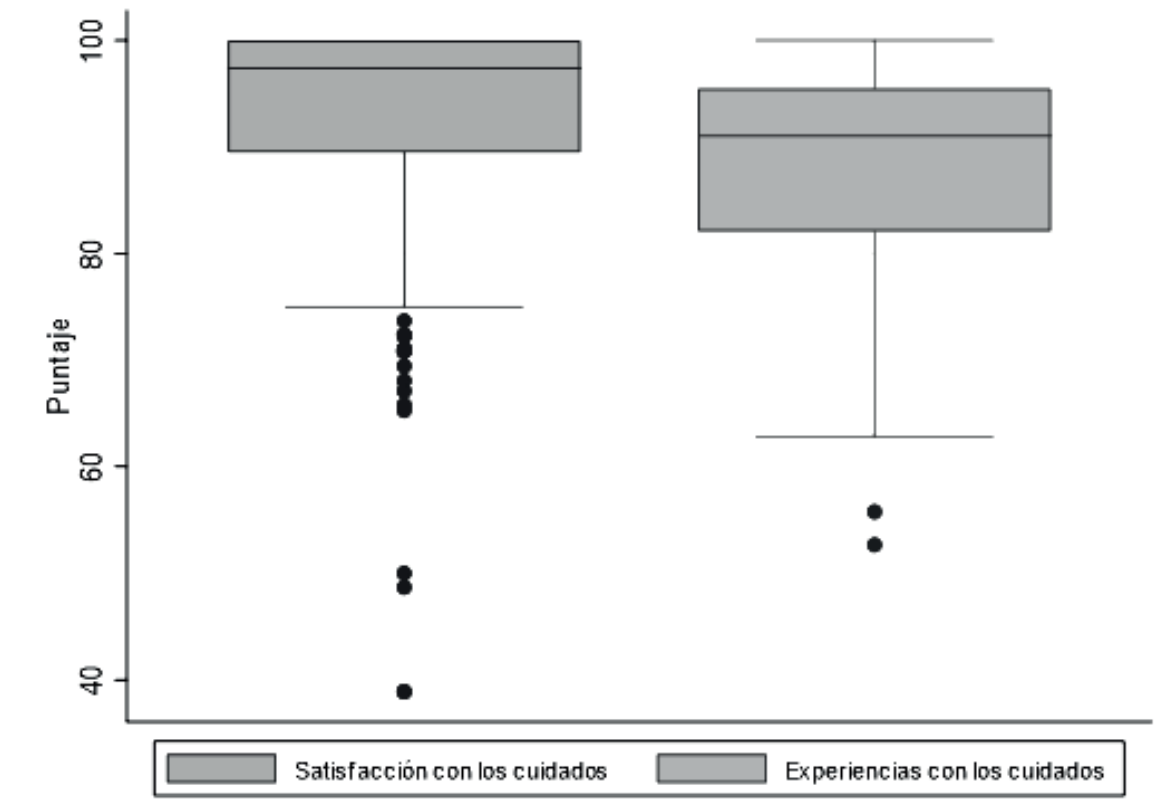

Figura 1. Gráfico de Cajas y bigotes de los puntajes en las dimensiones del Cuestionario de Calidad de Cuidados de Enfermería-CUCACE

Fuente: Diseño propio de los autores.
Se evidencia que los puntajes totales de cada una de las dos dimensiones del instrumento CUCACE, obtuvieron medias y medianas altas, indicando que los usuarios en general tuvieron una percepción positiva en la experiencia y satisfacción con los cuidados brindados por los estudiantes de enfermería. 


\section{Discusión}

El Consejo Internacional de Enfermería (CIE), resalta la importancia de cumplir con los cuatro deberes fundamentales de la profesión de enfermería: promover la salud, prevenir la enfermedad, restaurar la salud y aliviar el sufrimiento, de manera tal que se dignifique la vida, se humanice la prestación de los servicios y se ofrezca un cuidado de calidad ${ }^{30}$. Teniendo en cuenta los deberes de enfermería enunciados, este trabajo se convierte en uno de los primeros estudios en Colombia, en el que se investiga la calidad del cuidado desde la experiencia y satisfacción del usuario hospitalizado con los cuidados brindados por el estudiante de enfermería, a partir de la adaptación del cuestionario CUCACE como instrumento para la recolección de la información.

En el presente estudio la amabilidad del estudiante, el interés por el usuario, la cantidad y el tipo de información que le brindó acerca de la situación de salud y tratamiento y la capacitación del estudiante de enfermería para hacer bien su trabajo, fueron los ítems con puntuaciones altamente positivas; datos similares con los hallazgos de investigaciones desarrolladas en el ámbito internacional en estudiantes de enfermería en el entorno hospitalario; entre ellas el estudio de Oskay et al. ${ }^{14}$, el cual evaluó la satisfacción del paciente con la atención brindada por

En el presente estudio la amabilidad del estudiante, el interés por el usuario, la cantidad y el tipo de información que le brindó acerca de la situación de salud y tratamiento y la capacitación del estudiante de enfermería para hacer bien su trabajo, fueron los ítems con puntuaciones altamente positivas estudiantes de enfermería en una sala de perinatología, utilizando la escala Percepciones de la Calidad de la Atención de Enfermería - Forma abreviada (OPPQNCS-SF), las puntuaciones medias de las subescalas del instrumento alcanzaron los valores máximos, determinando que los estudiantes de enfermería lograron satisfacer las necesidades de los pacientes de una manera cuidadosa y atenta, proporcionando atención de acuerdo a los sentimientos del paciente, promovieron la comunicación entre otras enfermeras y pacientes, y brindaron cuidados de enfermería con conocimiento y habilidad.

Así mismo, los resultados reportados en el estudio desarrollado por Malekzadeh et al. ${ }^{15}$, demostraron que los pacientes se encuentran altamente satisfechos con la atención que recibieron de los estudiantes de enfermería en relación a las habilidades en comunicación, atención y educación; y Campiño et $a l^{13}$ en el que la categoría de apertura a la comunicación para proporcionar educación en salud a la persona presentó la puntuación más alta en la escala de siempre; los hallazgos de estos estudios son similares con los reportados en esta investigación, en la que se resaltan puntuaciones positivas en ítems como: explicación de los procedimientos, educación en relación a la situación de salud y tratamiento, la capacidad de escucha y la atención oportuna.

Por otra parte, Özer y Cetinkaya ${ }^{16}$ resaltan en los resultados de su investigación, que la mayoría de los pacientes estuvieron satisfechos con el cuidado, la información y el respeto que recibieron de los estudiantes de enfermería. Igualmente, en estudio de Can et al..$^{17}$ las puntuaciones más altas se encontraron en los ítems en que los pacientes estuvieron complacidos con el respeto que se les mostró, al recibir respuestas a sus preguntas, al mostrar interés sincero y al conocimiento de las enfermeras sobre su condición de salud; y el estudio de Campiño et $a l^{13}$ en el que la categoría de cualidades del hacer de enfermería, evidenció en sus respuestas un resultado principalmente en siempre y casi siempre. Los resultados de los estudios citados guardan similitud con esta investigación, en el que el conocimiento y la rapidez en el cuidado, el interés, el trato y el respeto hacia la persona, y la cantidad y el tipo de información brindada, 
presentaron puntuaciones altamente positivas.

Desde un enfoque cualitativo los estudios de Lindsey et al. ${ }^{18}$ y Eskilsson et al. ${ }^{19}$, puntualizaron los adjetivos más utilizados por los pacientes para describir su experiencia con la atención brindada por los estudiantes, entre ellos, divertido, maravilloso, preparado, servicial, atento, afectuoso, compasivo, cuidadoso sensible e informativo; otro aspecto recurrente en los comentarios de los pacientes fue el comportamiento profesional, amable y preocupado por los estudiantes de enfermería; adjetivos que se resalta en las dimensiones de experiencia y satisfacción de este trabajo.

El estudio de Elbasuony et al. ${ }^{31}$ sus resultados reflejan que la atención de enfermería brindada por los estudiantes de enfermería es deficiente y más de la mitad de las madres (63\%) no están satisfechas y tienen una actitud negativa hacia la participación de los estudiantes en su cuidado; contrario a los hallazgos de este estudio, en el que la percepción de los usuarios con la atención recibida por los estudiantes fue positiva en un $99.1 \%$.

Igualmente, se puede observar en el presente estudio la percepción y satisfacción positiva en los ítems relacionados con el tiempo que el estudiante dedicó al cuidado del paciente y la amabilidad del estudiante; resultados similares a los reportados por Suikkala et al. ${ }^{20}$ en el que se destaca como el conocimiento, las habilidades y la cantidad y calidad de tiempo son atributos necesarios en el estudiante hacia la construcción de relaciones interpersonales con el paciente; Topcu y Cakmak ${ }^{21}$, en el que los pacientes reconocieron el tiempo que invirtieron en ellos y los cuidados individualizados que recibieron por parte de los estudiantes de enfermería; Lindsey et al. ${ }^{18}$ en el que la amabilidad y la cortesía del estudiante de enfermería obtuvo el valor promedio más alto, también destacan la cantidad de tiempo que los estudiantes de enfermería pasaron con ellos y con sus familias enseñándoles a entender el proceso de la enfermedad.

Por otra parte, cabe mencionar que en el análisis de este estudio la dimensión de satisfacción destaca la percepción positiva en los ítems "el estudiante de enfermería le decía a la profesora los cuidados que estaba brindando" y"el estudiante de enfermería sabía que hacer sin depender de la profesora"; estos datos son similares a los resultados reportados por estudios de autores como Can et al. ${ }^{17}$ Eskilsson et al. ${ }^{19}$ y Suikkala et al. ${ }^{20}$ en donde se demostró que los pacientes destacan la presencia y supervisión de los profesores, instructores clínicos y enfermeras tituladas, como una manera de permitir la participación del estudiante, generar relaciones interpersonales y la sensación de seguridad del cuidado clínico recibido por el estudiante; por consiguiente la interacción entre estudiantes, profesores y pacientes juega un rol esencial en el estado de salud de los pacientes y mejora significativamente su nivel de satisfacción.

Contrastado los resultados de este estudio en los ítems"le fue fácil relacionarse con el estudiante de enfermería"y"percibió al estudiante de enfermería como un amigo o amiga", con los resultados del estudio de Suikkala et al. ${ }^{20}$, se encuentra que las relaciones interpersonales bilaterales entre los pacientes y los estudiantes, es una competencia necesaria para construir relaciones exitosas en la planificación del cuidado y en la educación al paciente y en la prestación de los servicios de salud.

Finalmente, este estudio permitió evaluar la satisfacción y experiencias del usuario con el cuidado brindado por estudiantes de enfermería. Sin embargo, presenta algunas limitaciones, una de ellas relacionada con la interacción del estudiante con la familia, la cual tan solo presenta un porcentaje de respuesta bajo, considerándose entre las razones el horario de ingreso de visita hospitalaria, la no presencia del familiar durante el tiempo de atención del estudiante y hasta la percepción del usuario en el actuar del estudiante. 
Los hallazgos de este estudio, sugieren a nivel nacional e internacional el desarrollo de nuevas investigaciones en los escenarios hospitalarios en los que el estudiante de enfermería lleva a cabo sus prácticas formativas, de manera que permita no solo la comparación de resultados, sino seguir corroborando el instrumento CUCACE como una herramienta válida y confiable para evaluar la satisfacción del paciente con el cuidado que recibe por el estudiante de enfermería.

\section{Conclusiones}

El alto nivel de percepción positiva en las dimensiones de experiencias y satisfacción de los usuarios con la atención brindada por los estudiantes de enfermería, significa que los estudiantes pueden satisfacer las necesidades y expectativas de cuidado de los usuarios, lo que puede servir como un indicador que apoya los estándares de calidad de la institución de salud.

Los resultados de este estudio servirán de base para la toma de decisiones, orientas a mejorar no solo la formación de un perfil profesional integral, competente y comprometido con la salud y de vida de la sociedad, sino con la calidad, el impacto y el reconocimiento académico del programa de enfermería.

Conflicto de intereses: Los autores declaran no tener ningún conflicto de intereses.

Financiación: Esta investigación es resultado de la Convocatoria interna de la Universidad Industrial de Santander sin financiación, identificada con código FS201708.

\section{Referencias}

1. República de Colombia. Decreto 1011 de 2006: por el cual se establece el sistema obligatorio de garantia de calidad de la atencion de salud del sistema general de seguridad social en salud. Ministerio de la Proteccion Social. Bogotá, Colombia: Diario Oficial 46.230; 2006.

2. República de Colombia. Ley 100 de 1993: por la cual se crea el sistema de seguridad social integral y se dictan otras disposiciones. Ministerio de Trabajo y Seguridad Social. Bogotá, Colombia: Diario Oficial 41.148; 1993.

3. República de Colombia. Ley 1122 de 2007: por la cual se hacen algunas modificaciones en el sistema general de seguridad social en salud y se dictan otras disposiciones. Congreso de la República de Colombia. Bogotá, Colombia: Diario Oficial 46.506; 2007.

4. República de Colombia. Ley 1438 de 2011: por medio del cual se reforma el Sistema General de Seguridad Social en Salud y se dictan otras disposiciones. Congreso de la República de Colombia. Bogotá, Colombia: Diario Oficial 47.957; 2011.

5. Lenis-Victoria CA, Manrique-Abril FG. Calidad del cuidado de enfermería percibida por pacientes hospitalizados. Aquichan. 2015;15(3):413-25.

http://dx.doi.org/10.5294/aqui.2015.15.3.9

6. Borré Ortiz YM, Vega Vega Y. Calidad percibida de la atención de enfermería por pacientes hospitalizados. Cienc. enferm. 2014; 20(3):81-94. http://dx.doi.org/10.4067/S0717-95532014000300008

7. Arciniegas Ipúz PA, Aya Aguilar LJ, Suarez Trujillo N, Salazar Herrán RL. La satisfacción en salud: el reflejo del profesionalismo, cuidado y acompañamiento emocional de Enfermería. Cina Research. 2018; 2(1):46-55.

https://journals.uninavarra.edu.co/index.php/cinaresearch/article/view/88/62 
8. Puch-Ku GF, Uicab-Pool GdIÁ, Ruiz-Rodríguez M, Castañeda-Hidalgo H. Dimensiones del cuidado de enfermería y la satisfacción del paciente adulto hospitalizado. Rev Enfer Inst Mex Seguro Soc. 2016; 24(2):129-36.

https://www.medigraphic.com/pdfs/enfermeriaimss/eim-2016/eim162i.pdf

9. Marín Laredo M, Álvarez Huante C, Valenzuela Gandarilla J, Dimas Palacios C. Calidad percibida de la atención de enfermería en usuarios hospitalizados. Estudio Comparativo. Revista de Técnicas de la Enfermería. 2017; 1(1):9-16. https://www.ecorfan.org/republicofperu/research_journals/Revista_de_Tecnicas_de_la_Enfermeria/vol 1 num 1/Revista_de_T\%C3\%A9cnicas_de_la_Enfermeria_y_Salud_V1_N1.pdf

10.Ramírez Perdomo CA, Perdomo Romero AY, Galán González EF. Evaluación de la calidad del cuidado de enfermería en la unidad de cuidados intensivos. Av. enferm. 2014; 31(1):42-51. http://www.scielo.org.co/pdf/aven/v31n1/v31n1a05.pdf

11.Freitas JSd, Silva AEBdC, Minamisava R, Bezerra ALQ, Sousa MRGd. Quality of nursing care and satisfaction of patients attended at a teaching hospital. Rev Latino- Am. Enfermagem. 2014; 22(3):454-60. https://doi.org/10.1590/0104-1169.3241.2437

12.González D. Percepción de comportamientos de cuidado humanizado en estudiantes de enfermería. Rev. actual enf. 2014; 17(2): 14-21. https://encolombia.com/medicina/revistas-medicas/enfermeria/ve-172/percepcion-comportamientos-humanizado/

13.Campiño SM, Duque PA, Cardozo VH. Percepción del paciente hospitalizado sobre el cuidado brindado por estudiantes de enfermería. Univ. Salud. 2019; 21(3):215-225. http://dx.doi.org/10.22267/rus.192103.158

14.Oskay U, Güngor I, Basgöl S. Evaluation of Patients' Satisfaction With Nursing Students' Care on a Perinatology Ward. J Nurs Educ. 2015;54(12):696-703.

https://doi.org/10.3928/01484834-20151110-06

15.Malekzadeh J, Amouzeshi Z, Mazlom SR. A quasi-experimental study of the effect of teaching Orem's self-care model on nursing students' clinical performance and patient satisfaction. Nurs Open. 2018;5(3):370-5. https://doi.org/10.1002/nop2.151

16.Özer N, Çetinkaya F. Satisfacción de pacientes que viven en clínicas quirúrgicas de enfermeras de estudiantes. Revista Anadolu de Enfermería y Ciencias de la Salud. 2010;13(1):5865. https://dergipark.org.tr/tr/download/article-file/29510

17. Can G, Akin S, Aydiner A, Ozdilli K, Durna Z. Evaluation of the effect of care given by nursing students on oncology patients' satisfaction. Eur J Oncol Nurs. 2008; 12(4):387-92. https://doi.org/10.1016/j.ejon.2008.02.004

18. Lindsey DL, Henly SJ, Tyree EA. Outcomes in an academic nursing center: client satisfaction with student services. J Nurs Care Qual. 1997; 11(5):30-8.

https://doi.org/10.1097/00001786-199706000-00008

19.Eskilsson C, Carlsson G, Ekebergh M, Hörberg U. The experiences of patients receiving care from nursing students at a Dedicated Education Unit: A phenomenological study. Nurse Educ Pract. 2015; 15(5):353-8. doi: https://doi.org/10.1016/j.nepr.2015.04.001

20.Suikkala A, Koskinen S, Leino-Kilpi H. Patients' involvement in nursing students' clinical education: A scoping review. Int J Nurs Stud. 2018; 84:40-51.

https://doi.org/10.1016/j.ijnurstu.2018.04.010

21.Topcu SY, Cakmak B. Surgical Patients' Perceptions of the Care Provided by Student Nurses. International Journal of Caring Sciences. 2014;7(1):195-202. https://www.researchgate.net/ publication/281438592_Surgical_Patients'_Perceptions_of_the_Care_Provided_by_Student_Nurses

22. Universidad Industrial de Santander, Escuela de Enfermería. Proyecto Educativo Programa de Enfermería. Bucaramanga. Bucaramanga, Colombia.2012. http://www.uis.edu.co/webUIS/es/academia/facultades/salud/escuelas/enfermeria/presentacion.jsp 
23.StataCorp LP. Stata Statistical Sofware: Release 12.1. Collage Station, Texas: Stata Corporation; 2011.

24.Torres Contreras C, Buitrago-Vera M. Percepción de la calidad del cuidado de enfermería en pacientes oncologicos hospitalizados. Rev Cuid. 2011;2(2):138-48.

https://doi.org/10.15649/cuidarte.v2i1.49

25.Alonso R, Blanco-Ramos MÁ, Gayoso P. Validación de un cuestionario de calidad de cuidados de enfermería. Rev Calid Asist. 2005;20(5):246-50.

https://doi.org/10.1016/S1134-282X(05)75092-2

26.Thomas LH, McColl E, Priest J, Bond S, Boys RJ. Newcastle satisfaction with nursing scales: an instrument for quality assessments of nursing care. Qual Health Care. 1996;5(2):67-72.

https://doi.org/10.1136/qshc.5.2.67

27. Torres Contreras CCT, Páez Esteban AN, Rincón Sepúlveda L, Rosas Baez D, Mendoza Moreno EP. Reproducibilidad del cuestionario: calidad de cuidados de enfermería en pacientes hospitalizados. Rev Cuid. 2016;7(2):1338-44.

doi: http://dx.doi.org/10.15649/cuidarte.v7i2.339

28.República de Colombia. Resolución No008430: por la cual se establecen las normas científicas, técnicas y administrativas para la investigación en salud. Colombia. Ministerio de Salud. Bogotá, Colombia. 1993.

29.República de Colombia. Ley 911 de 2004: por la cual se dictan disposiciones en materia de responsabilidad deontológica para el ejercicio de la profesión de Enfermería en Colombia, se establece el régimen disciplinario correspondiente y se dictan otras disposiciones. Congreso de la República. Bogotá, Colombia: Diario Oficial No. 45.693; 2004.

30.De Arco-Canoles O, Suarez-Calle ZK. Rol de los profesionales de enfermería en el sistema de salud colombiano. Univ Salud. 2018;20(2):171-82.

doi: http://dx.doi.org/10.22267/rus.182002.121

31.Elbasuony M, Raju J, R A C, Abdallah N. Maternal Attitude and Satisfaction towards Involvement of Nursing Students in their Care. Int J Sci Res (Raipur). 2019;8(2):86-90. https://doi.org/10.21275/ART20194939 